\title{
Childhood and adolescent trauma in Pietermaritzburg is a neglected sub-epidemic within a larger epidemic and warrants a dedicated service
}

\author{
B Conradie, ${ }^{1}$ (i) W Xu, ${ }^{1}$ (i) V Kong, ${ }^{2}$ (D) DL Clarke,,${ }^{2,3}$ (D) JL Bruce, ${ }^{3}$ (D) V Manchev, ${ }^{2,3}$ (i) GL Laing, ${ }^{3}$ (i) W Bekker ${ }^{3}$ (i) \\ ${ }^{1}$ Department of Surgery, University of Auckland, New Zealand \\ ${ }^{2}$ Department of Surgery, University of the Witwatersrand, South Africa \\ ${ }^{3}$ Department of Surgery, University of KwaZulu-Natal, South Africa
}

Corresponding author, email: bernikeconradie@gmail.com

Background: This study reviews our experience with paediatric trauma to help plan and strengthen ongoing strategies to deal with trauma in our region.

Methods: All children and adolescents less than 18 years of age who were admitted to our centre following trauma between December 2012 and October 2020 were reviewed.

Results: Over the 8-year period, 2091 children were admitted with trauma to Grey's Hospital. There were $1479(71 \%)$ male patients, median age: 10 years. One thousand four hundred and fifty-eight $(70 \%)$ patients were referral from rural regions. In $1597(76 \%)$ patients, the mechanism of injury was blunt trauma. A total of $387(19 \%)$ patients underwent a surgical intervention, whilst 1641 (78\%) patients were managed non-operatively. In 63 patients, management records were missing. Multiple patients required multiple surgical procedures. A total of 144 patients had a laparotomy, 70 a soft tissue debridement procedure, 40 an orthopaedic procedure, 53 a neurosurgical procedure, which included 37 patients who underwent craniotomy, and 18 patients underwent a fasciotomy for compartment syndrome. There were 82 patients who required miscellaneous procedures. A total of $213(10 \%)$ patients required ICU admission. There were $48(2.3 \%)$ deaths. Conclusion: The volume of child and adolescent trauma managed is significant and suggests that a dedicated paediatric trauma service in the region is warranted. It is hoped that future partnering with appropriate stakeholders will allow this service to continue to mature and provide leadership in all aspects of care of injured children in the region.

Keywords: multiple trauma, general surgery, paediatrics

\section{Introduction}

South Africa continues to experience a disproportionate, refractory burden of trauma for a country with such relative political stability. ${ }^{1,2}$ In many ways, trauma in South Africa is a neglected epidemic. However, within this epidemic itself, there are well defined vulnerable populations with specific risks and needs who tend to be overlooked. These are the hidden sub-epidemics within the larger epidemic itself and the vulnerable groups include women, children and adolescents, the elderly and those with mental health care issues. These vulnerable groups warrant more detailed study and merit specific targeted programmes and services as they experience a degree of double jeopardy due to being both under-resourced and under-researched. . $^{3-6}$

The Lancet Commission on Global Surgery (LCOGS) has highlighted the fact that there is both a disproportionate burden of surgical diseases in low- and middle-income countries of the world in comparison to the high-income countries and in resources to manage this burden. ${ }^{7}$ South Africa is a middle-income country with major historical racialised inequalities in wealth and access to health care. ${ }^{1}$ Trauma in children and adolescents has not been the subject of the same intense focus that adult trauma has in South Africa. The trauma systems approach, which was pioneered by the American College of Surgeons has been adopted in South Africa. Outside of the Western Cape and Gauteng Province the focus has been on adult trauma care in adult centres. ${ }^{8-11}$ KwaZulu-Natal Province has a large youthful population but has no dedicated children's institution. In addition, there has been little involvement in trauma care by dedicated paediatric surgeons or intensivists. The drive to develop a paediatric trauma service has mostly come from adult trauma surgeons. ${ }^{12-16}$ Trauma in children and adolescents is relatively under-researched in comparison to other forms of trauma.

A growing research interest in a topic usually precedes and motivates efforts to address the problem, as was the case with the massive increase in research into global surgery topics over the last two decades. This paper reviews an 8-year experience with the care of children and adolescents who have sustained trauma in the city of Pietermaritzburg and the western third of the province. The aim of the project is to describe and document the burden and outcome of childhood and adolescent trauma in the region. It is hoped that this will contribute to addressing the paucity of research 


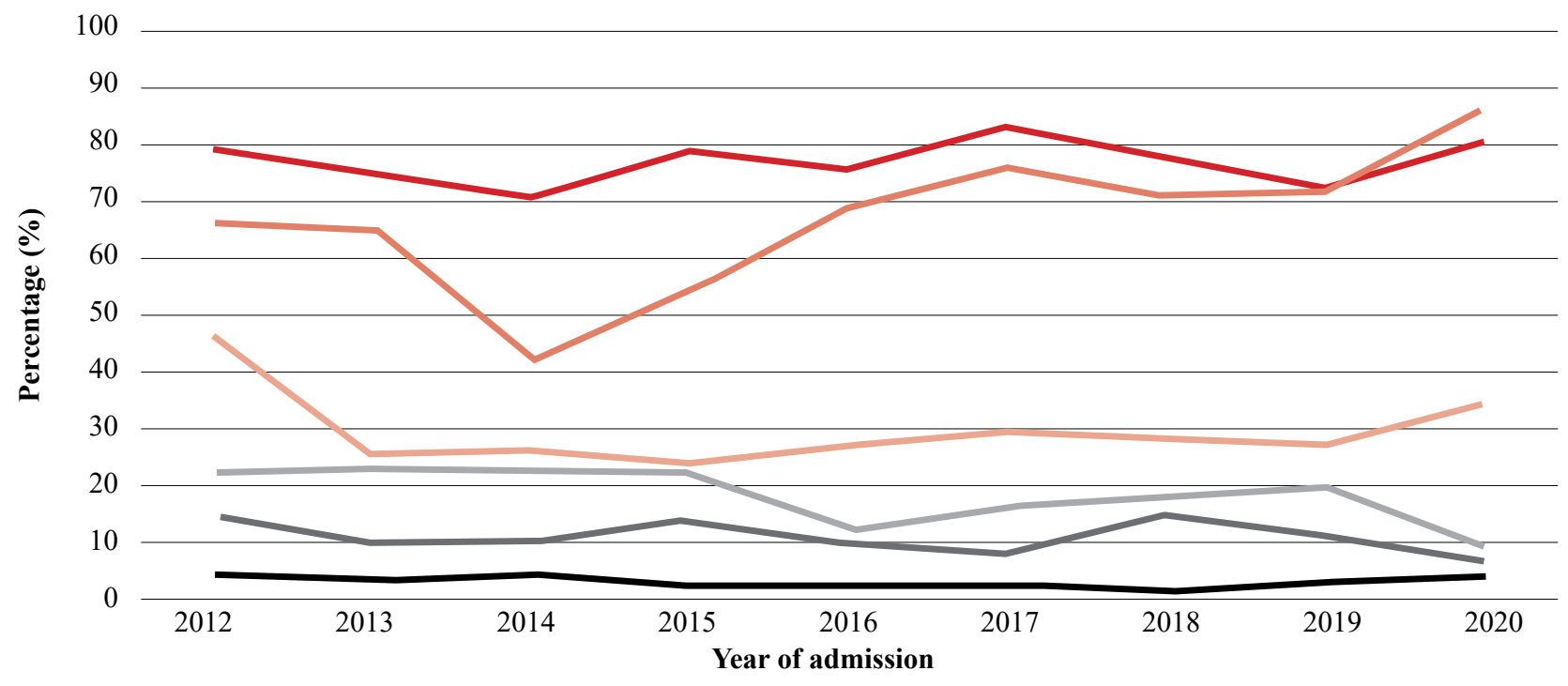

- Blunt trauma - ICU $=$ Operative management $=$ Morbidity experienced $=$ CT scan Died

Figure 1: Management and outcomes stratified by year across an 8-year period

on the topic and provide evidence to sustain and nurture ongoing efforts to develop a paediatric trauma service in the region.

\section{Methods}

\section{Setting}

The Pietermaritzburg Metropolitan Trauma Service (PMTS) is based at Grey's Hospital, which is a general tertiary care public hospital located in Pietermaritzburg, the capital of KwaZulu-Natal (KZN) Province, South Africa. The hospital serves as the referral centre for 19 district and three regional hospitals within the western part of the province. The western third of the province has a population of three million people and many of the regions score badly in terms of markers of social deprivation.

Injured children and adolescents are taken to the nearest facility, which is usually a district hospital. Basic assessment and stabilisation take place at these institutions. Patients are referred through to the PMTS based on severity and complexity. There is an aero-medical and a road-based retrieval system available. The PMTS consists of four dedicated attending surgeons and a team of house staff. All trauma resuscitations and operations are supervised by the attending staff. There is a team of three paediatric surgeons who focus on elective work and non-trauma childhood emergencies. Their assistance may be enlisted as required. There is a dedicated cohort of paediatric anaesthesiologists who supervise paediatric anaesthetics and a dedicated paediatric ICU of four beds and an adult ICU capable of caring for children over the age of ten. There is a radiology service with a single CT scanner. Within the last two years, a nascent emergency department has been created with specialist emergency physicians, and emergency sonography has been introduced. A single neurosurgeon has recently been appointed. There is no cardiothoracic service and no vascular surgery service available.

The PMTS maintains a prospectively entered surgical registry known as the hybrid electronic medical registry (HEMR). ${ }^{14}$ Ethics approval for the HEMR has been granted by the Biomedical Research Ethics Committee of the University of KwaZulu-Natal (ref. no. BE207/09 BCA 221/13). This approval is renewed annually and is current. The system has been functional since December 2012.

\section{Design}

All children younger than 18 years of age who were admitted by the PMTS following trauma between December 2012 and October 2020 were included. Patient demographics, mechanism of injury, management strategy (operative or non-operative), complications, patient residential status (urban or rural), hospital and intensive care duration of stay, and 30-day mortality rates were extracted. A surgical procedure was defined as an invasive procedure requiring a trip to the operating room. Procedures which occurred outside of the OR were not generally recorded. Burn victims are treated by a dedicated burn service and were excluded from this analysis. Body region injuries were graded using the abbreviated injury scale (AIS) which is a 6-point scoring system to determine the severity of traumatic injury to that body region. An AIS of 1 would refer to minor injury, AIS 4 to severe with AIS 6 being maximal/untreatable.

\section{Statistical analysis}

All relevant data were extracted and initially summarised onto an Excel spreadsheet for review. All statistical analysis was performed using SPSS version 19 (IBM Corp. Released 2010. IBM SPSS Statistics for Windows, Version 19.0. Armonk, NY: IBM Corp.) and R (version 4.0.2; R Foundation for Statistical Computing, Vienna, Austria). ${ }^{17}$ Patients were stratified by AAST Organ Injury Scale Grade. All normally distributed continuous variables were described using means with standard deviation (SD). Continuous variables with gross skewness were reported using a median with interquartile range (IQR). Mann-Whitney U and chisquare tests were used to compare skewed continuous and categorical data respectively between operative and nonoperative groups. Non-parametric (asymmetrical) data were descriptively described. 


\section{Results}

Over the 8-year period, 2091 children and adolescents were admitted to Grey's Hospital following trauma. There were $1479(71 \%)$ boys and the rest were girls. The median age was 10 years $(0-18)$ and $1458(70 \%)$ patients were of rural origin. In 1597 (76\%) patients, the mechanism of injury was blunt trauma. The median age for blunt trauma was 9 years old and 15 for penetrating trauma. In 455 this was in the form of an accidental fall from height (median age of 7 years), in 413 a pedestrian-vehicle accident (PVA) with a median age of 8 years, in 306 a motor vehicle accident (MVA) with a median age of 12 years, in 274 an assault (median age 15 years), in 42 a collapse of a structure, in 19 an attempted hanging, and in 16 an animal injury. In 27 cases the mechanism was not specified. A total of 475 (23\%) patients sustained penetrating trauma. These included $156 \mathrm{stab}$ wounds (median age 17 years old), 141 animal bites (median age 7 years), 125 gunshot wounds (median age 16 years old), 28 impalements, and 16 assaults with miscellaneous weapons. In nine patients, the mechanism was not specified. The remainder sustained either a miscellaneous spectrum of injuries, such as swallowing a piece of glass, or did not have a complete history recorded. This data showed that children who received penetrating injuries tended to be older, and there were age differences in the different mechanisms of injuries.

\section{Imaging}

A total of 1354 (65\%) patients had a computed tomography scan (CT). These included 977 CT scans of the head, 262 of the abdomen, 146 of the whole body, 110 of the pelvis, 115 of the thorax, and 17 of an extremity. A total of 105 patients had a CT angiogram, and 77 patients had a sonographic investigation.

\section{Body regions injured}

A total of $1369(65 \%)$ patients had an injury to their head and neck, $406(19 \%)$ an abdominal injury, 601 (29\%) patients an injury to an extremity, $352(17 \%)$ an injury to the thorax, $141(7 \%)$ an injury to the pelvis, $353(17 \%)$ a maxillofacial injury and 25 patients a perineal injury. The median AIS for abdominal injury was 3, median AIS for maxillofacial injury was 1 . Median AIS for injury to the head and neck was 2 . Median AIS for external injury was 2, median AIS for injury to extremity was 2, and median AIS for injury to chest was also 2 .

\section{Management}

A total of 387 (19\%) patients underwent at least one surgical intervention, whilst $1641(78 \%)$ patients were managed non-operatively. Multiple patients required multiple surgical procedures. A total of 144 patients had a laparotomy and 70 a soft tissue debridement procedure. Forty (40) patients had an orthopaedic procedure, 53 a neurosurgical procedure, which included 37 patients who underwent craniotomy, and 18 patients underwent a fasciotomy for compartment syndrome. There were 82 patients who required miscellaneous procedures. Sixty-two (62) patients (3\%) required additional surgery after their initial procedure. This included 29 patients who underwent a repeat laparotomy. Four patients needed two repeat laparotomies and three required more than two repeat laparotomies.

\section{Splenic trauma}

Over the eight-year period, $66(3 \%)$ patients sustained splenic trauma, of which $48(72 \%)$ were male, and median age was 12 years (0-18 years). Sixty-one (93\%) sustained blunt trauma. Forty-nine (49) patients $(78 \%)$ had an AAST grade 3 or less splenic injury and $14(22 \%)$ had an AAST grade 4 or 5 injury. In three patients, AAST grade of the splenic injury was not recorded. Only seven patients $(11 \%)$ had an isolated splenic injury, while the remaining 59 (89\%) had other associated injuries. Forty-five (45) patients $(68 \%)$ were managed non-operatively, whilst the remainder were subjected to laparotomy. Five (7\%) required a splenectomy and one required angio-embolisation. All seven patients with isolated splenic injury were managed non-operatively. Four (6\%) patients died. All four non-survivors had significant traumatic brain injury (TBI) as well as significant respiratory compromise. Three of the four died secondary to their TBI and one patient died as a result of a splenic injury.

\section{Renal trauma}

Over the eight-year period, a total of $63(3 \%)$ children sustained renal trauma. Of these 63 patients, 16 (25\%) were female and the median age was 13 years $(0-18$ years). Thirtyone (31) patients $(49 \%)$ were of rural origin. In $57(90 \%)$ patients, the mechanism of injury was blunt trauma. Eight (13\%) patients had an AAST I injury; seven (11\%) an AAST II kidney injury; 17 (27\%) an AAST III injury; 19 (30\%) had an AAST IV injury; and nine (14\%) had AAST V injury. In three patients, the AAST grade was not documented. In $26(41 \%)$ patients, the renal injury was isolated whilst the remainder had other additional abdominal injuries.

Forty-five (45) of the 63 patients $(71 \%)$ were managed non-operatively. The remaining $18(29 \%)$ patients underwent a laparotomy and five $(8 \%)$ required a nephrectomy. Of the 26 patients with isolated kidney injuries, only three required an operation. Two (3\%) patients died. One of these was managed non-operatively, and the other operatively. Neither underwent a nephrectomy. The non-operative patient had profound TBI, whilst the other developed ischaemic bowel.

\section{Hepatic trauma}

Over the eight-year period, 75 (4\%) children sustained liver trauma; $53(71 \%)$ male and $22(29 \%)$ female. The median age was 10 years old. There were eight $(10 \%)$ penetrating mechanisms of injury. The median grade of liver injury was 2 (range 1-5). Grade of liver injuries based on imaging included: 16 patients (24\%) with grade I, 21 (28\%) with grade II, $19(25.3 \%)$ with grade III, ten $(13 \%)$ with grade IV, and five $(6.7 \%)$ with grade $\mathrm{V}$ injuries.

Twenty-three (31\%) children underwent laparotomy; six $(8 \%)$ of which required damage control surgery (DCS). Three (4\%) children died, two having undergone a laparotomy. Two of the deaths were following hypovolaemic shock and one was due to ischaemic bowel (same patient as is mentioned in renal trauma).

\section{Overall outcomes}

A total of $213(10 \%)$ patients required ICU admission. There were $48(2.3 \%)$ deaths. A total of $384(18 \%)$ of patients required ventilation during their stay. Figure 1 summarises the trend in admission, management and outcomes in the cohort over the 8-year period - this shows that there has 
been a very consistent trend in all parameters over the 8 years. Of the 48 deaths, ten $(21 \%)$ were admitted to the ICU, and $12(25 \%)$ underwent an operation (seven laparotomies, three tracheostomies and two others). Forty $(83 \%)$ required ventilation. Five $(10 \%)$ of these patients required blood transfusion. Thirty-eight $(80 \%)$ of these patients sustained blunt trauma, while the remainder sustained penetrating injuries. The median admission Glasgow Coma Scale (GCS) in the non-survivors was 4.5, whereas it was 15 in the survivors.

\section{Discussion}

The World Health Organization (WHO) describes childhood injuries as a global public health problem. Our data support this statement, with approximately 22 children and adolescents requiring admission each month to our institution. Each admission requires access to considerable resources such as imaging, access to the operating room and intensive care. In addition, apart from the not insignificant mortality rate associated with child and adolescent trauma, there is also major lifelong morbidity associated with this disease. This is particularly egregious as for every child fatality, there are many more children who survive with a chronic disability. This chronic disability is associated with ongoing poverty as it impacts on educational achievement and subsequent ability to achieve employment. In addition, poor families experience both the initial catastrophic medical expense and then compounding chronic expenses, which detract from the family's ability to access other resources such as nutrition, education and sanitation..$^{2,3}$

The response to paediatric trauma must be a systemic one. The development of trauma systems with a dedicated paediatric trauma centre to provide leadership in all aspects of trauma care has been shown in North America to improve outcomes. Whilst this approach has been adopted by adult trauma surgeons in South Africa, there has been less uptake in the paediatric surgical community. In KZN, there is no dedicated children's hospital or paediatric trauma service. ${ }^{5,6,8-10,18}$ Our data shows consistent trends across the 8 -year period, suggesting that a dedicated paediatric trauma service is needed for the region.

There is a high rate of penetrating trauma in this cohort. This is cause for concern as penetrating trauma is overwhelmingly interpersonal in nature and is more likely to require surgical intervention than blunt trauma. ${ }^{16,19}$ Injury prevention programmes to reduce interpersonal violence and penetrating trauma cannot rely on mechanical and law enforcement strategies exclusively. These programmes require complex social interventions, which must address concerns around social behaviour and the need for affirmation amongst boys and young men. ${ }^{11}$

The need for operation translates into greater use of resources as evidenced by the much higher percentage of ICU stay accorded to postoperative patients. However, the successful management of blunt trauma also requires access to resources as evidenced by the high rate of CT scans in these patients. A particular area where there is a lag in uptake in technology is in the relatively low usage of sonar in our cohort. Clinician performed sonar has become ubiquitous in North America and this has resulted in a decrease in the use of CT scan in children. ${ }^{20}$ This technological lag is an example of the discrepancy in health resources which the LCOGS has highlighted. Mortality is associated with the severity of the overall trauma as well as with TBI, blunt trauma and hypothermia. ${ }^{1}$

\section{Conclusion}

The volume of child and adolescent trauma managed by the PMTS is significant and suggests that a dedicated paediatric trauma service in the region is warranted. It is hoped that future partnering with appropriate stakeholders will allow this service to continue to mature and provide leadership in all aspects of care of injured children in the region.

\section{Conflict of interest}

The authors declare no conflict of interest.

\section{Funding source}

No funding was required.

\section{Ethical approval}

Ethical approval for the HEMR has been granted by the Biomedical Research Ethics Committee of the University of KwaZulu-Natal (ref. no. BE207/09 BCA 221/13). This approval is renewed annually and is current.

\section{ORCID}

B Conradie (iD https://orcid.org/0000-0002-9141-1467

W Xu (iD https://orcid.org/0000-0002-1270-2769

V Kong (iD https://orcid.org/0000-0003-2291-2572

DL Clarke (iD https://orcid.org/0000-0002-6954-153X

JL Bruce (iD https://orcid.org/0000-0001-8666-4104

V Manchev (iD https://orcid.org/0000-0001-9152-0092

GL Laing (iD https://orcid.org/0000-0001-8075-0386

W Bekker (iD https://orcid.org/0000-0003-0695-5994

\section{REFERENCES}

1. Buitendag JJ, Kong VY, Bruce JL, et al. The spectrum and outcome of paediatric traumatic brain injury in KwaZuluNatal Province, South Africa has not changed over the last two decades. S Afr Med J. 2017;107(9):777-80. https://doi. org/10.7196/SAMJ.2017.v107i9.12394.

2. Meara JG, Leather AJ, Hagander L, et al. Global Surgery 2030: Evidence and solutions for achieving health, welfare, and economic development. Lancet. 2015;386(9993):569624. https://doi.org/10.1016/S0140-6736(15)60160-X.

3. Mock C, Abantanga F, Goosen J, Joshipura M, Juillard C. Strengthening care of injured children globally. Bull World Health Organ. 2009;87(5):382-9. https://doi.org/10.2471/ BLT.08.057059.

4. St-Louis E, Séguin J, Roizblatt D, et al. Systematic review and need assessment of pediatric trauma outcome benchmarking tools for low-resource settings. Pediatr Surg Int. 2017;33(3):299-309. https://doi.org/10.1007/s00383-0164024-9.

5. Bradshaw CJ, Bandi AS, Muktar Z, et al. International study of the epidemiology of paediatric trauma: PAPSA Research Study. World J Surg. 2018;42(6):1885-94. https://doi. org/10.1007/s00268-017-4396-6.

6. Herbert HK, Van As AB, Bachani AM, et al. Patterns of pediatric injury in South Africa: an analysis of hospital data between 1997 and 2006. J Trauma Acute Care Surg. 2012;73(1):16874. https://doi.org/10.1097/TA.0b013e31824d67c3. 
7. Meara JG, Hagander L, Leather AJM. Surgery and global health: a Lancet Commission. Lancet. 2014;383(9911):12-3. https://doi.org/10.1016/S0140-6736(13)62345-4.

8. Metcalfe D, Bouamra O, Parsons NR, et al. Effect of regional trauma centralization on volume, injury severity and outcomes of injured patients admitted to trauma centres. Br J Surg. 2014;101(8):959-64. https://doi.org/10.1002/bjs.9498.

9. Mooney DP, Gutierrez IM, Chen Q, Forbes PW, Zurakowski D. Impact of trauma system development on pediatric injury care. Pediatr Surg Int. 2013;29(3):263-8. https://doi. org/10.1007/s00383-012-3232-1.

10. Wesson HKH, Bachani AM, Mtambeka P, et al. Changing state of pediatric injuries in South Africa: An analysis of surveillance data from a Pediatric Emergency Department from 2007 to 2011. Surgery. 2017;162(6):S4-11. https://doi. org/10.1016/j.surg.2017.03.019.

11. Isaac KN, Van Niekerk A, Van As AB. Child road traffic crash injuries at the Red Cross War Memorial Children's Hospital in Cape Town, South Africa in 1992, 2002 and 2012. Int J Inj Contr Saf Promot. 2015;22(4):352-8. https://doi.org/10.1080/ 17457300.2014 .912236

12. Manchev V, Bruce JL, Oosthuizen GV, Laing GL, Clarke DL. The incidence, spectrum and outcome of paediatric trauma managed by the Pietermaritzburg Metropolitan Trauma Service. Ann R Coll Surg Engl. 2015;97(4):274-8. https://doi. org/10.1308/003588414X14055925061595.

13. Traynor MD, Hernandez MC, Shariq O, et al. Trauma registry data as a tool for comparison of practice patterns and outcomes between low- and middle-income and high-income healthcare settings. Pediatr Surg Int. 2019;35(6):699-708. https://doi. org/10.1007/s00383-019-04453-w.
14. Laing GL, Bruce JL, Skinner DL, et al. Development, implementation, and evaluation of a hybrid electronic medical record system specifically designed for a developing world surgical service. World J Surg. 2014;38(6):1388-97. https:// doi.org/10.1007/s00268-013-2438-2.

15. Donovan MM, Kong VY, Bruce JL, et al. The hybrid electronic medical registry allows benchmarking of quality of trauma care: a five-year temporal overview of the trauma burden at a major trauma centre in South Africa. World J Surg. 2019;43(4):1014-21. https://doi.org/10.1007/s00268-018-048 80-1.

16. Khumalo-Mugabi L, Moffatt S, Bekker W, et al. Penetrating trauma in children and adolescents in Pietermaritzburg. S Afr J Surg. 2020;58(1):33-6. https://doi.org/10.17159/20785151/2020/v58n1a3017.

17. A language and environment for statistical computing [Internet]. Vienna, Austria; 2013. Available from: http:// www.r-project.org.

18. Lutge E, Moodley N, Tefera A, et al. A hospital based surveillance system to assess the burden of trauma in KwaZuluNatal Province South Africa. Injury. 2016;47(1):135-40. https://doi.org/10.1016/j.injury.2015.08.020.

19. Clarke DL, Allorto NL, Thomson SR. An audit of failed non-operative management of abdominal stab wounds. Injury. 2010;41(5):488-91. https://doi.org/10.1016/j.injury.20 09.10.022.

20. Traynor MD, Zielinski MD, Moir CR, et al. CT scans for pediatric injury in a middle-income country trauma center: are we repeating past mistakes? J Pediatr Surg. 2021;56(12):23427. https://doi.org/10.1016/j.jpedsurg.2021.01.035. 